\section{Violência contra a criança: revelando o perfil dos atendimentos em serviços de emergência, Brasil, 2006 e 2007}

\author{
Violence against children: revealing the \\ characteristics of emergency treatment, Brazil, \\ 2006 and 2007
}

\author{
1 Secretaria de Vigilância em \\ Saúde, Ministério da Saúde, \\ Brasília, Brasil. \\ 2 Universidade Federal do \\ Piauí, Teresina, Brasil. \\ 3 Universidade Federal de \\ Minas Gerais, Belo Horizonte, \\ Brasil. \\ 4 Universidade Federal de \\ Goiás, Goiânia, Brasil. \\ 5 Secretaria Municipal de \\ Saúde, Curitiba, Brasil. \\ Correspondência \\ M. D. M. Mascarenhas \\ Coordenação Geral de \\ Vigilância de Doenças e \\ Agravos Não Transmissíveis, \\ Secretaria de Vigilância em \\ Saúde, Ministério da Saúde. \\ SAF Sul, Trecho 02, Lotes 05 e \\ 06, Bl. F, Torre I, Ed. Premium, \\ Sl-14, Brasília, DF \\ 70070-600, Brasil. \\ mdm.mascarenhas@gmail. \\ com
}

\begin{abstract}
The aim of this article was to describe the profile of emergency care for injuries resulting from violence against children (<10 years of age) treated Surveillance System for Violence and Accidents (VIVA) of the Ministry of Health, for 30 consecutive days in 2006 and 2007 in selected cities of Brazil. The 518 children in the sample were predominantly male (60.6\%), aged 5-9 years (52.1\%), and black (71.2\%). The majority (55\%) of the cases occurred in the home, 34.2\% involved sharp objects, and $68.7 \%$ evolved to discharge. The most frequent form of violence was physical aggression (67.4\%), involving beating, sharp objects, and firearms. The other types of violence included neglect (32\%), psychological abuse (9.5\%), and sexual assault (3.5\%). The aggressors were largely male (48.1\%) and family members of the victim (36.3\%). The study shows children's vulnerability to situations of violence. It is necessary to develop specific strategies for inter-sector care and social mobilization to intervene in this problem.
\end{abstract}

Violence; Child; Emergency Medical Services

\author{
Márcio Dênis Medeiros Mascarenhas 1,2 \\ Deborah Carvalho Malta 1,3 \\ Marta Maria Alves da Silva 1,4 \\ Cheila Marina Lima 1,4 \\ Mércia Gomes Oliveira de Carvalho 1 \\ Vera Lídia Alves de Oliveira ${ }^{5}$
}

\section{Introdução}

A violência é, atualmente, um dos maiores desafios impostos às autoridades de saúde pública em todo o planeta, pois aflige a humanidade sob diversas formas e em diferentes cenários. Estima-se que, no mundo, mais de um milhão de pessoas perdem a vida em decorrência da violência a cada ano, sem considerar o contingente de vítimas não fatais 1 . As lesões decorrentes da violência afetam pessoas de todas as idades, mas parecem causar mais danos entre as crianças, que pertencem a um dos grupos mais vulneráveis na sociedade 2,3 .

Independentemente dos termos usados para nomeá-la, a violência contra as crianças está representada em toda ação ou omissão capaz de provocar lesões, danos e transtornos a seu desenvolvimento integral. Tais eventos geralmente envolvem uma relação assimétrica e desigual de poder manifestada pela força física, pelo poder econômico ou político, pela dominação e opressão familiar ou geracional 4 .

A violência contra a criança pode ocorrer sob diversas manifestações, em diferentes grupos econômicos e sociais, sendo essencial sua compreensão para o melhor entendimento das estratégias de promoção à saúde e prevenção. O tipo mais descrito na literatura tem sido a violência intrafamiliar, que se caracteriza por todo tipo de omissão que prejudique o bem-estar, a integridade física, psicológica, a liberdade, ou direito de 
desenvolvimento de algum membro da família 5 . Porém, outras formas de violência ainda incluem a violência estrutural, em decorrência da estrutura social e das desigualdades produzidas, que pode se manifestar nos espaços públicos, atingindo meninos e meninas de rua, em reformatórios e abrigos, ou produzidas contra meninos e meninas trabalhadores 6,7. Outro tipo de violência, a escolar, ou bullying, manifesta-se por ameaças e humilhações entre escolares 5 .

O tema da violência contra a criança tem sido cada vez mais objeto de estudos no país e no mundo, além de ter, nas últimas décadas, ocupado, cada vez mais, as agendas da saúde pública 1,2,3. Segundo a Organização Mundial da Saúde (OMS) ${ }^{8}$, podem ser distinguidos quatro tipos de violência contra a criança: abuso físico, sexual, emocional ou psicológico e negligência, os quais podem resultar em danos físicos, psicológicos; danos ao crescimento, desenvolvimento e maturação das crianças. Na maioria das vezes esses efeitos sequer podem ser mensurados, resultando em danos irreversíveis nessas crianças. Muitas vezes esses eventos permanecem silenciosos e silenciados no interior das famílias, escolas, comunidade e serviços de saúde 5 .

Uma pequena parcela desses eventos pode ser dimensionada por meio das estatísticas de mortalidade e morbidade. Segundo levantamento realizado pela OMS 2, estima-se que, a cada ano, violências e acidentes correspondam a mais de 950 mil mortes de crianças menores de 18 anos de idade, além de causar mais de 10 milhões de incapacidades. A maioria dessas mortes refere-se a causas evitáveis e consideradas não intencionais, como: acidentes de trânsito, afogamentos, queimaduras, quedas. Além dessas causas não intencionais, consideram-se ainda as violências intencionais, como as violências físicas e sexuais, que respondem por mais de 200 mil mortes por ano entre jovens e crianças 2 .

No Brasil, segundo panorama apresentado pelo Ministério da Saúde (http://www.datasus. gov.br, acessado em 30/Jun/2009), em 2006 foram registrados 310 homicídios entre crianças menores de dez anos de idade, o que representa $7 \%$ do total de mortes por causas externas neste grupo etário. No mesmo ano, o risco de morte por agressões fatais foi de $0,9 / 100$ mil menores de dez anos, variando de 0,7 a 1,0/100 mil entre meninas e meninos, respectivamente. Todavia, ao se estratificar os indicadores segundo faixa etária, verificou-se que crianças menores de um ano estiveram expostas a maior risco de morte por violência (2,3/100 mil) quando comparadas às de 1 a 4 anos (0,6/100 mil) e de 5 a 9 anos $(0,8 / 100$ mil). Quanto à morbidade por lesões decorrentes da violência, foram hospitalizadas 2.226 crianças menores de 10 anos (2,3\% do total de internações por causas externas) na rede própria ou conveniada do Sistema Único de Saúde (SUS). A taxa bruta de internação foi de 6,1/100 mil, variando de 4,4/100 mil entre as meninas a 7,8/100 mil entre os meninos. Segundo a faixa etária, as maiores taxas de hospitalização foram observadas entre os menores de um ano (7,0/100 mil), variando de 5,2/100 mil meninas a 8,7/100 mil meninos. Entre os meninos de 5 a 9 anos, observou-se a maior taxa hospitalização: 9,1/100 mil.

Diversas fontes de dados contribuem para a construção do conhecimento da magnitude da violência contra as crianças 9 . Dentre elas, destacam-se os registros de denúncias aos conselhos tutelares e delegacias especializadas 10,11,12, os estudos sobre casos atendidos em serviços especializados 13,14,15 e as análises da concepção de profissionais de saúde acerca desta problemática 16,17 . No entanto, apesar das variadas fontes de informação atualmente existentes no Brasil e em diferentes partes do mundo, nenhuma delas é capaz de, isoladamente, descrever a totalidade da extensão do problema, principalmente nos aspectos referentes às lesões não fatais e de menor gravidade, o que requer o desenvolvimento de novas estratégias para preencher esta lacuna 18 .

Assim, a partir de experiências internacinais e considerando as especificidades brasileiras, o Ministério da Saúde implantou, a partir de 2006, o Sistema de Vigilância de Violências e Acidentes (VIVA), que se destina a descrever o perfil dos atendimentos às vítimas de causas externas realizados em serviços de urgência e emergência do SUS. Trata-se de uma estratégia de vigilância que pretende contribuir com o conhecimento acerca da distribuição, magnitude e tendência desses agravos para balizar o planejamento e aplicação de intervenções apropriadas de prevenção 19

O presente estudo tem como objetivo descrever as características epidemiológicas dos atendimentos de emergência por lesões relacionadas às causas violentas em crianças menores de dez anos de idade, atendidas em serviços que integraram o VIVA nos anos de 2006 e 2007.

\section{Métodos}

Realizou-se estudo descritivo com dados obtidos na pesquisa sobre atendimentos por causas externas (acidentes e violências) em unidades de urgência e emergência da rede própria do SUS, em capitais de estados e municípios selecionados, que compõem o VIVA. Os dados foram coletados em plantões alternados de 12 horas durante 30 dias consecutivos, entre os meses de setembro 
a novembro de 2006 (65 serviços em 34 municípios e Distrito Federal) e 2007 (84 serviços em 36 municípios e Distrito Federal). O aumento dos serviços e de municípios se deu em função da expansão do VIVA em serviços sentinelas de referência de outros municípios e capitais que não realizaram a coleta de dados no ano de 2006.

Foram consideradas elegíveis para a realização da pesquisa as capitais das Unidades Federadas, sendo que Porto Alegre (Rio Grande do Sul), São Paulo e Belém (Pará) não participaram por problemas operacionais. Além disso, foram incluídas outras cidades que atenderam aos seguintes critérios: elevado coeficiente de morbimortalidade por causas externas, existência de serviços de referência para o atendimento às vítimas de violências e acidentes, execução de ações intersetoriais de prevenção das violências e acidentes. As demais cidades foram distribuídas nos estados de São Paulo, Pernambuco e Espírito Santo. Maiores detalhes da metodologia podem ser vistos em publicação específica 19 .

Foram entrevistadas todas as vítimas de acidentes ou violência que procuraram atendimento nos serviços de emergência selecionados no período da pesquisa. Adotou-se a definição de violência apresentada pela OMS 1: uso da força física ou do poder, real ou em ameaça, contra si próprio, contra outra pessoa, ou contra um grupo ou uma comunidade, que resulte ou tenha qualquer possibilidade de resultar em lesão, morte, dano psicológico, deficiência de desenvolvimento ou privação. Considerou-se acidente todo evento não intencional e evitável, causador de lesões físicas e emocionais, no âmbito doméstico ou nos outros ambientes sociais como o do trabalho, trânsito, escola, esporte ou lazer, de acordo com as diretrizes da Política Nacional de Redução da Morbimortalidade por Acidentes e Violências, do Ministério da Saúde 20.

A cada paciente admitido no setor de emergência em decorrência de causas externas (acidente e violências), os entrevistadores iniciavam a abordagem da vítima ou acompanhante (quando o paciente era menor ou encontrava-se impossibilitado de responder) para solicitar autorização e iniciar a entrevista. Utilizou-se formulário elaborado pelo Ministério da Saúde para obter informações sobre: aspectos demográficos (idade, sexo, raça/cor, escolaridade), meio de transporte utilizado para chegar ao hospital, tipo de ocorrência (acidente ou violência), local de ocorrência (residência, escola, via pública, trabalho, bar, local de prática de esporte), tipo de acidente (acidente de transporte, queda, queimadura, dentre outros), tipo de violência (agressões, maus-tratos, suicídio), características do provável autor da agressão (sexo, relação com a vítima), localização anatômica da lesão e desfecho do atendimento (alta, hospitalização, óbito).

Os dados foram coletados por entrevistadores previamente treinados por técnicos (coordenadores locais) das secretarias de saúde dos municípios selecionados, os quais, por sua vez, haviam sido capacitados pela equipe de coordenadores nacionais da pesquisa na sede do Ministério da Saúde. Durante o treinamento da equipe de coordenadores locais, apresentamos a operacionalização do VIVA; seleção dos serviços de urgência e emergência; padronização das definições adotadas; uniformização das técnicas de obtenção, registro e fluxo de envio dos dados. Todos os formulários foram digitados no nível local e enviados para o Ministério da Saúde para compor o banco de dados nacional.

No presente estudo, foram selecionados os registros referentes aos atendimentos de emergência por lesões decorrentes de violências envolvendo crianças menores de dez anos de idade. Devido à baixa freqüência observada, foram excluídos os registros de atendimento por tentativa de suicídio $(n=7)$. Os dados foram processados no programa Epi Info 3.5.1 (Centers for Disease Control and Prevention, Atlanta, Estados Unidos), comparando-se as freqüências dos atendimentos registrados no período de estudo segundo as características da vítima (sexo, idade, faixa etária) e da ocorrência (local de ocorrência, natureza da lesão, evolução). Os atendimentos por violência foram classificados segundo a tipologia proposta pela OMS 8: abuso físico, sexual, emocional ou psicológico, negligência. Apresentamos a prevalência e os respectivos intervalos de 95\% de confiança dos diferentes tipos de violência segundo algumas características da vítima, da ocorrência e do provável autor da agressão.

O projeto foi aprovado pela Comissão Nacional de Ética em Pesquisa (CONEP), tendo sido garantido anonimato e privacidade aos pacientes, profissionais e gestores dos serviços onde o estudo foi realizado. A assinatura do termo de consentimento livre e esclarecido foi substituída pelo consentimento verbal, obtido pelo paciente ou por seu responsável. No caso de menores de dez anos, objeto deste estudo, o consentimento verbal e a coleta de informações foram obtidos junto ao acompanhante (familiar ou responsável).

\section{Resultados}

Durante as pesquisas realizadas em 2006 e 2007, foram registrados 106.075 atendimentos devidos a causas externas (acidentes e violências) nos serviços de urgência e emergência selecionados. 
Os eventos violentos foram responsáveis por $10.610(10 \%)$ atendimentos, dos quais 519 (5\%) corresponderam às vítimas menores de dez anos de idade.

Dentre as principais características das crianças atendidas, salientamos os seguintes aspectos: predomínio de vítimas do sexo masculino (razão $\mathrm{M} / \mathrm{F}=1,5)$, faixa etária de 5 a 9 anos $(52,2 \%)$ e cor de pele negra $(71,1 \%)$. A residência $(55,1 \%)$ foi o principal cenário para a ocorrência de violência, seguida de espaços públicos $(17,9 \%)$ e escola $(17,3 \%)$. As lesões mais freqüentes incluíram corte/perfuração (34,1\%), contusão (14,3\%), entorse/luxação $(8,3 \%)$ e fratura $(6,9 \%)$. Em relação à evolução, a maior parcela das vítimas $(68,8 \%)$ recebeu alta logo após o atendimento de emergência, $27,6 \%$ foram hospitalizadas ou transferidas para outros serviços. Ocorreram cinco óbitos dentro das primeiras 24 horas após o atendimento de emergência inicial. As maiores diferenças entre meninos e meninas foram observadas nos atendimentos cujas vítimas tinham de 5 a 9 anos (razão $\mathrm{M} / \mathrm{F}=1,9$ ), possuíam pele negra (razão $\mathrm{M} / \mathrm{F}=1,6$ ), encontravam-se em via pública (razão $M / F=2,9$ ) no momento da ocorrência e que apresentaram lesões mais graves como corte/ perfuração (razão M/F = 2,2) (Tabela 1).

Segundo a tipologia do evento, percebe-se que a violência física $(42,2 \%)$ foi a principal forma de abuso contra as crianças, apresentando freqüência maior entre os meninos (razão $\mathrm{M} / \mathrm{F}=$ 1,6). Dentre os meios utilizados para efetuar esse tipo de violência, destacou-se o espancamento/ força física, atingindo 33,7\%. A negligência (32\%) foi o segundo tipo de violência identificado entre os atendimentos envolvendo crianças menores de dez anos, apresentando distribuição semelhante entre meninos e meninas (razão $\mathrm{M} / \mathrm{F}=$ $1,1)$. Os abusos do tipo psicológico $(9,4 \%)$ e sexual $(3,5 \%)$ apresentaram as menores proporções de ocorrência, porém foram mais freqüentes entre as vítimas do sexo feminino (razão $\mathrm{M} / \mathrm{F}=0,8$ e 0,1, respectivamente) (Tabela 2).

Entre as meninas, os indivíduos identificados como prováveis autores da agressão foram, em sua maioria, os próprios familiares da vítima (46,8\%). Para os meninos, pessoas de convívio próximo das vítimas, mas sem laços de parentesco direto, foram os agressores mais freqüentes $(37,6 \%)$, seguidos por familiares da vítima (29,6\%) e por aqueles totalmente desconhecidos (10,5\%). A agressão perpetrada por conhecido não familiar e por desconhecido foi duas vezes maior entre os meninos em comparação com as meninas (razão $\mathrm{M} / \mathrm{F}=2,0$ ). Em quase $50 \%$ do total de vítimas, predominou o agressor do sexo masculino, principalmente entre os meninos (razão $M / F=2,2$ ). Porém, foi possível perceber que as meninas eram vítimas de agressores do sexo feminino em maior freqüência do que os meninos (razão M/F = 0,6) (Tabela 2).

Com exceção da negligência, os demais tipos de violência apresentaram tendência de aumento proporcional à idade das vítimas. Abuso físico foi observado em $47,7 \%$ das crianças menores de um ano, chegando a apresentar freqüência próxima de $90 \%$ entre as crianças a partir de sete anos de idade. De maneira inversa, a negligência apresentou freqüência máxima entre as crianças com até dois anos de idade $(71,1 \%)$, passando a apresentar tendência de declínio até atingir a proporção de $8,3 \%$ entre as vítimas de nove anos de idade. Abusos de ordem psicológica e sexual mantiveram-se em proporção inferior a 15\% em todas as idades, atingindo a freqüência máxima de $13,3 \%$ e $8,3 \%$, respectivamente, entre as crianças com nove anos de idade (Figura 1).

A Tabela 3 apresenta a prevalência dos diferentes tipos de violência segundo as principais características das vítimas, da ocorrência e do provável autor de violência contra as crianças. A violência física teve maior prevalência entre os meninos (43\%), crianças de 5 a 9 anos (50,9\%), brancos $(45,5 \%)$, na via pública $(54,8 \%)$, com agressores conhecidos $(55,1 \%)$ e do sexo masculino (52,6\%). A ocorrência de negligência predominou entre vítimas do sexo feminino $(38,5 \%)$, menores de um ano (56\%), de cor negra $(32,2 \%)$, na residência (50,3\%), tendo por agressor o familiar $(48,1 \%)$. As vítimas de violência psicológica e sexual apresentaram prevalências semelhantes, sendo observados os maiores valores entre as vítimas do sexo feminino, com idades de 5 a 9 anos, de cor branca, com ocorrência na residência e predomínio de agressor do sexo masculino. Entre as vítimas de violência psicológica, prevaleceu o familiar (14,3\%) como o principal agressor, enquanto o desconhecido $(8,2 \%)$ foi o principal agressor entre as vítimas de violência sexual.

\section{Discussão}

A violência contra a criança consiste em matéria identificada e discutida ao longo da história da civilização ocidental. O tema vem ganhando importância nas agendas e compromissos internacionais e nacionais $1,2,20,21,22$. Mudanças culturais têm ocorrido no sentido de valorizar a criança e de diminuir o poder dos pais sobre ela, o que leva à mudança da percepção da violência como algo natural e um direito legitimamente exercido pelos pais sobre a criança. A promulgação do Estatuto da Criança e Adolescente, a implantação dos Conselhos Tutelares, a organização dos sistemas locais de saúde e a criação dos serviços de pre- 
Distribuição dos atendimentos de emergência por violência em menores de dez anos, por sexo, segundo características da vítima e da ocorrência. Municípios selecionados, Brasil, 2006-2007.

\begin{tabular}{|c|c|c|c|c|c|c|c|}
\hline \multirow[t]{2}{*}{ Características } & \multicolumn{2}{|c|}{ Masculino (M) } & \multicolumn{2}{|c|}{ Feminino (F) } & \multicolumn{2}{|c|}{ Total } & \multirow{2}{*}{$\begin{array}{c}\text { Razão } \\
\text { M/F }\end{array}$} \\
\hline & $\mathbf{n}$ & $\%$ & $\mathrm{n}$ & $\%$ & $\mathrm{n}$ & $\%$ & \\
\hline \multicolumn{8}{|l|}{ Faixa etária (anos) } \\
\hline$<1$ & 49 & 15,6 & 51 & 24,9 & 100 & 19,3 & 1,0 \\
\hline $1-4$ & 88 & 28,0 & 60 & 29,3 & 148 & 28,5 & 1,5 \\
\hline $5-9$ & 177 & 56,4 & 94 & 45,9 & 271 & 52,2 & 1,9 \\
\hline \multicolumn{8}{|l|}{ Raça/cor da pele } \\
\hline Negra (preta + parda) & 226 & 72,0 & 143 & 69,8 & 369 & 71,1 & 1,6 \\
\hline Branca & 77 & 24,5 & 55 & 26,8 & 132 & 25,4 & 1,4 \\
\hline Outras & 6 & 1,9 & 1 & 0,5 & 7 & 1,3 & 6,0 \\
\hline Sem informação & 5 & 1,6 & 6 & 2,9 & 11 & 2,1 & \\
\hline \multicolumn{8}{|l|}{ Local de ocorrência } \\
\hline Residência & 155 & 49,4 & 131 & 63,9 & 286 & 55,1 & 1,2 \\
\hline Via pública & 69 & 22,0 & 24 & 11,7 & 93 & 17,9 & 2,9 \\
\hline Escola & 57 & 18,2 & 33 & 16,1 & 90 & 17,3 & 1,7 \\
\hline Outros & 25 & 8,0 & 9 & 4,4 & 34 & 6,6 & 2,8 \\
\hline Sem informação & 8 & 2,5 & 8 & 3,9 & 16 & 3,1 & \\
\hline \multicolumn{8}{|l|}{ Natureza da lesão } \\
\hline Corte/Perfuração & 122 & 38,9 & 55 & 26,8 & 177 & 34,1 & 2,2 \\
\hline Contusão & 42 & 13,4 & 32 & 15,6 & 74 & 14,3 & 1,3 \\
\hline Entorse/Luxação & 23 & 7,3 & 20 & 9,8 & 43 & 8,3 & 1,2 \\
\hline Fratura & 22 & 7,0 & 14 & 6,8 & 36 & 6,9 & 1,6 \\
\hline Outra & 78 & 24,8 & 60 & 29,3 & 138 & 26,6 & 1,3 \\
\hline Sem lesão & 18 & 5,7 & 17 & 8,3 & 35 & 6,7 & 1,1 \\
\hline Sem informação & 9 & 2,9 & 7 & 3,4 & 16 & 3,1 & \\
\hline \multicolumn{8}{|l|}{ Evolução * } \\
\hline Alta & 216 & 68,8 & 141 & 68,8 & 357 & 68,8 & 1,5 \\
\hline Internado/Transferido & 89 & 28,3 & 54 & 26,3 & 143 & 27,6 & 1,6 \\
\hline Óbito & 2 & 0,6 & 3 & 1,5 & 5 & 1,0 & 0,7 \\
\hline Sem informação & 7 & 2,2 & 7 & 3,4 & 14 & 2,7 & \\
\hline Total & 314 & 100,0 & 205 & 100,0 & 519 & 100,0 & 1,5 \\
\hline
\end{tabular}

* Foi observada a evolução dos casos durante as primeiras 24 horas após o atendimento de emergência inicial.

Fonte: Sistema de Serviços Sentinelas de Vigilância de Violências e Acidentes (VIVA), Ministério da Saúde.

venção de violência são ações que contribuem para o aumento dos registros e da comunicação da violência infantil 22,23,24,25. Além disso, a perspectiva de resposta social ao problema estimula a comunicação individual e coletiva das situações de maus-tratos. A "lei do silêncio" vem lentamente sendo quebrada 26 . Assim, reforça-se a necessidade de aumento e aprofundamento nos estudos sobre violência contra a criança, como a análise dos dados aqui apresentados.

O presente estudo mostra o predomínio do sexo masculino entre as vítimas desses eventos, o que pode ser justificado pelos diferentes comportamentos de cada sexo e por fatores culturais, que determinam maior liberdade aos meninos e, em contrapartida, maior vigilância sobre as meninas. Nesse aspecto, a maior ocorrência de agressões entre os meninos pode ser relacionada com um dos grandes símbolos de masculinidade no mundo atual, as armas, que materializam o poder de submeter o outro a seus desejos e interesses, poder de vida ou morte, por meio de objetos que são introduzidos desde cedo na vida do menino, na forma de brinquedos, e passam a fazer parte do universo masculino 27 .

O ambiente domiciliar foi o local em que grande parte dos eventos violentos tomou lugar, sendo este o ambiente favorável para a ocorrência de agressões e abusos contra crianças, principalmente as meninas. Por permanecerem mais tem- 
Figura 1

Proporção de atendimentos de emergência por violência em menores de dez anos segundo tipo de violência e idade da vítima. Municípios selecionados, Brasil, 2006-2007.

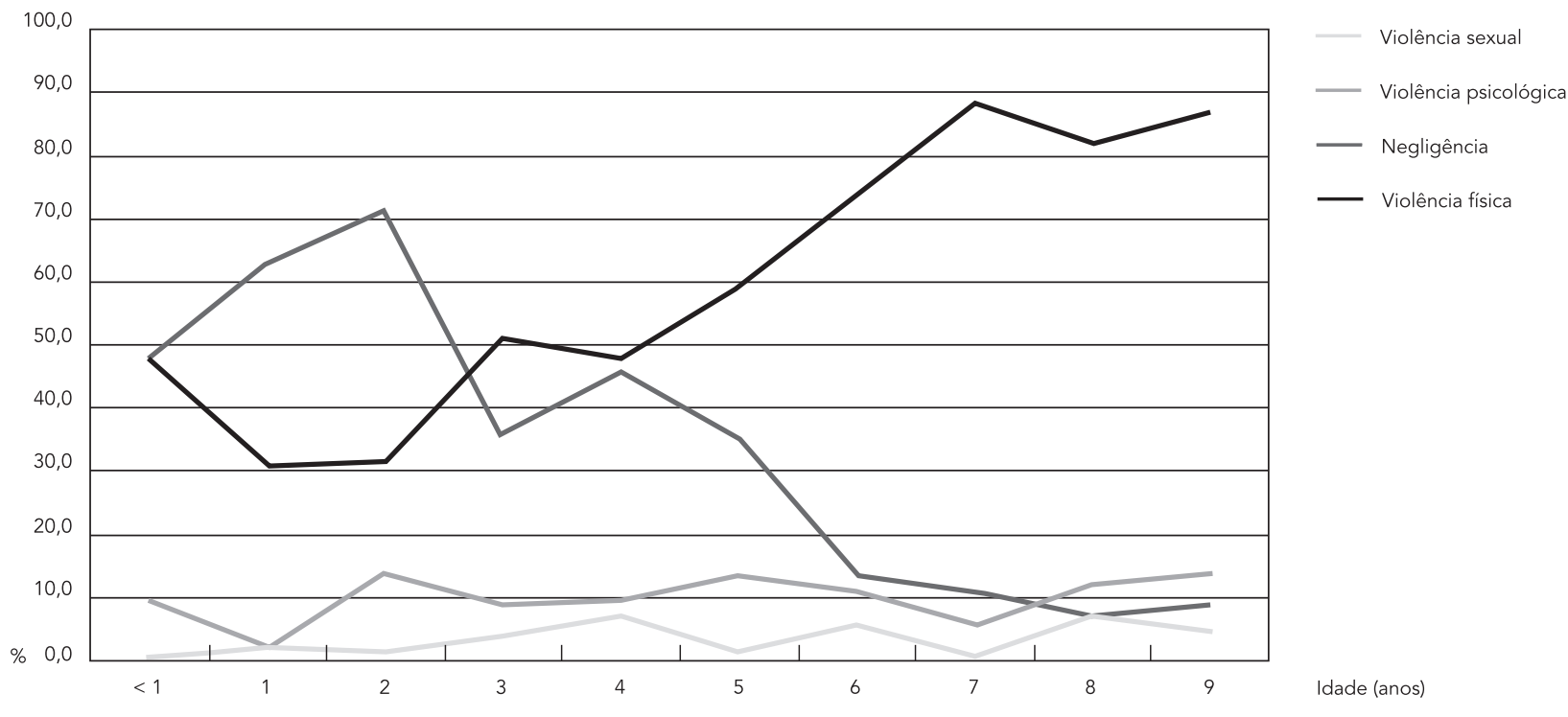

po em seus lares, as crianças acabam sendo violentadas mais freqüentemente nestes locais 10 .

A literatura 1,2,6,10,14 aponta as meninas como as maiores vítimas de violência sexual, negligência, prostituição, enquanto os meninos são as maiores vítimas de agressões físicas. Os tipos de agressões registrados no presente estudo apresentam distribuição diferente segundo o sexo da vítima, mostrando que os meninos sofrem maiores agressões físicas e as meninas mais agressões sexuais. Verificou-se predomínio de abuso físico e negligência/abandono, este um importante problema social, caracterizado pela omissão ou ato de deixar de prover as necessidades e cuidados básicos para o desenvolvimento físico, emocional e social da pessoa - o abandono é uma forma extrema de negligência. Esses achados são semelhantes aos resultados obtidos em Guarulhos, Estado de São Paulo 28.

Qualquer membro da família pode se tornar, em determinadas circunstâncias, vítima ou autor de violência. Entretanto, as crianças, por serem os mais indefesos e dependentes na família e na sociedade, são as principais vítimas desse tipo de violência, tanto na extensão como na gravidade dos danos e suas conseqüências 29 . A literatura assinala também os espaços públicos (ruas, bares e outros locais públicos) como importante cenário para a ocorrência de eventos violentos, especialmente quando envolvem agressores e vítimas do sexo masculino, enquanto que no espaço privado do lar ocorre a maioria das violências contra as meninas 27 .

A evolução do paciente após o atendimento de emergência pode ser empregada como reflexo da gravidade da lesão. Dessa forma, os resultados do presente estudo permitem estimar uma pequena proporção de casos graves, haja vista a menor freqüência de atendimentos cujo desfecho foi o óbito ou internação hospitalar em relação aos atendimentos que evoluíram para alta ou acompanhamento ambulatorial. Outros estudos sobre violência na infância também destacam a menor gravidade dos casos 30,31 .

Entretanto, o perfil de ocorrências pode variar conforme a fonte ou local pesquisado. No caso de hospitais de urgência e emergência, ou pesquisas realizadas com base em fontes do setor saúde, os maus-tratos físicos tendem a ocupar o primeiro posto entre as modalidades de violência provavelmente por serem mais graves e exigirem atendimento de saúde 14,16. Estudos conduzidos no ambiente escolar ou a partir de notificação do Conselho Tutelar tendem a detectar outras situações e destacam a negligência com principal ocorrência 10,11,32. 
Distribuição dos atendimentos de emergência por violência em menores de dez anos, por sexo, segundo tipo de violência e provável autor da agressão. Municípios selecionados, Brasil, 2006-2007.

\begin{tabular}{|c|c|c|c|c|c|c|c|}
\hline \multirow[t]{2}{*}{ Características } & \multicolumn{2}{|c|}{ Masculino (M) } & \multicolumn{2}{|c|}{ Feminino (F) } & \multicolumn{2}{|c|}{ Total } & \multirow{2}{*}{$\begin{array}{c}\text { Razão } \\
\text { M/F }\end{array}$} \\
\hline & $\mathrm{n}$ & $\%$ & $\mathrm{n}$ & $\%$ & $\mathbf{n}$ & $\%$ & \\
\hline \multicolumn{8}{|l|}{ Tipo de violência * } \\
\hline Física & 135 & 43,0 & 84 & 41,0 & 219 & 42,2 & 1,6 \\
\hline Espancamento & 103 & 32,8 & 72 & 35,1 & 175 & 33,7 & 1,4 \\
\hline Perfurocortante & 26 & 8,3 & 10 & 4,9 & 36 & 6,9 & 2,6 \\
\hline Arma de fogo & 10 & 3,2 & 6 & 2,9 & 16 & 3,1 & 1,7 \\
\hline Negligência & 87 & 27,7 & 79 & 38,5 & 166 & 32,0 & 1,1 \\
\hline Psicológica & 22 & 7,0 & 27 & 13,2 & 49 & 9,4 & 0,8 \\
\hline Sexual & 2 & 0,6 & 16 & 7,8 & 18 & 3,5 & 0,1 \\
\hline Outro & 3 & 1,0 & - & - & 3 & 0,6 & - \\
\hline \multicolumn{8}{|c|}{ Relação do provável autor da agressão com a vítima } \\
\hline Familiar & 93 & 29,6 & 96 & 46,8 & 189 & 36,4 & 1,0 \\
\hline Conhecido & 118 & 37,6 & 58 & 28,3 & 176 & 33,9 & 2,0 \\
\hline Desconhecido & 33 & 10,5 & 16 & 7,8 & 49 & 9,4 & 2,1 \\
\hline Sem informação & 70 & 22,3 & 35 & 17,1 & 105 & 20,2 & 2,0 \\
\hline \multicolumn{8}{|c|}{ Sexo do provável autor da agressão } \\
\hline Masculino & 170 & 54,1 & 79 & 38,5 & 249 & 48,0 & 2,2 \\
\hline Feminino & 47 & 15,0 & 74 & 36,1 & 121 & 23,3 & 0,6 \\
\hline Ambos & 17 & 5,4 & 9 & 4,4 & 26 & 5,0 & 2,1 \\
\hline Sem informação & 80 & 25,5 & 43 & 21,0 & 123 & 23,7 & 1,9 \\
\hline Total & 314 & 100,0 & 205 & 100,0 & 519 & 100,0 & 1,5 \\
\hline
\end{tabular}

* Não totaliza 100\%, pois era permitido informar mais de um tipo de violência para a mesma vítima.

Fonte: Sistema de Serviços Sentinelas de Vigilância de Violências e Acidentes (VIVA), Ministério da Saúde.

Estudo realizado em Feira de Santana, Bahia, baseando-se em registros do Conselho Tutelar, identificou a negligência como a violência mais prevalente, seguida de violência física, a violência psicológica e por último, a sexual. Em menores de um ano, registrou-se considerável ocorrência de negligência, violência física e psicológica. A violência sexual foi registrada em todas as faixas etárias, com maior prevalência acima dos dez anos, e a exploração sexual, a partir dos 14 anos 10. Pesquisas realizadas com base em dados do setor educacional também detectaram a negligência e falta de controle parental como a principal ocorrência. Tal constatação pode ser explicada por serem mais perceptíveis ou valorizadas pelos educadores, frente às mudanças de comportamento das crianças 32 . Além disso, a negligência se destaca em crianças menores de dois anos e reduz entre as maiores, o que pode ser explicado por ser a faixa na qual as crianças mais dependem dos adultos, para os cuidados.

$\mathrm{O}$ atual estudo detectou o crescimento da violência física com o aumento da idade, o que também foi identificado por Faleiros et al. 32 e por
Gomes et al. 14, e que foi atribuído ao desenvolvimento e crescimento das crianças, intensificando atitudes e comportamentos decorrentes da sua situação de violência, quando os responsáveis passam a realizar mais ameaças e punições corporais. O estudo atual apresentou menor freqüência de vítimas entre os menores de um ano de idade, embora seja nesta faixa etária que se observam as maiores taxas de mortalidade por violência 1,2 (http://www.datasus.gov.br, acessado em 30/Jun/2009).

Com relação ao autor do evento violento, destaca-se a maior participação de agressores conhecidos não familiares quando a vítima era do sexo masculino. Para as vítimas do sexo feminino, o agressor era, predominantemente, um membro da família. Esses resultados podem ser explicados pela maior ocorrência de violência contra meninos nos espaços públicos, onde estão envolvidos os agressores em relações extrafamiliares com a vítima, enquanto o espaço privado do domicílio é mais propício para a ocorrência de violência contra a mulher 10,27. 
Número de crianças (n), freqüência (f), prevalência (p) e intervalo de 95\% de confiança (IC95\%) dos atendimentos de emergência, por tipo de violência, segundo características da vítima, local da ocorrência e provável autor da agressão. Municípios selecionados, Brasil, $2006-2007$.

\begin{tabular}{|c|c|c|c|c|c|c|c|c|c|}
\hline \multirow[t]{3}{*}{ Características } & \multirow[t]{3}{*}{$\mathbf{n}$} & \multicolumn{8}{|c|}{ Tipo de violência $(n=519$ *) } \\
\hline & & \multicolumn{2}{|c|}{ Física } & \multicolumn{2}{|c|}{ Negligência } & \multicolumn{2}{|c|}{ Psicológica } & \multicolumn{2}{|c|}{ Sexual } \\
\hline & & $f$ & p (IC95\%) & $f$ & p (IC95\%) & $f$ & p (IC95\%) & $f$ & p (IC95\%) \\
\hline \multicolumn{10}{|l|}{ Sexo } \\
\hline Masculino & 314 & 135 & $43,0(37,4-48,7)$ & 87 & $27,7(22,9-33,1)$ & 22 & $7,0(4,5-10,6)$ & 2 & $0,6(0,1-2,5)$ \\
\hline Feminino & 205 & 84 & $41,0(34,2-48,0)$ & 79 & $38,5(31,8-45,6)$ & 27 & $13,2(8,9-18,6)$ & 16 & $7,8(4,5-12,4)$ \\
\hline \multicolumn{10}{|l|}{ Faixa etária (anos) } \\
\hline$<1$ & 100 & 36 & $36,0(26,6-46,2)$ & 56 & $56,0(45,7-65,9)$ & 5 & $5,0(1,6-11,3)$ & 1 & $1,0(0,0-5,4)$ \\
\hline $1-4$ & 148 & 45 & $30,4(23,1-38,5)$ & 73 & $49,3(41,0-57,7)$ & 15 & $10,1(5,8-16,2)$ & 6 & $4,1(1,5-8,6)$ \\
\hline $5-9$ & 271 & 138 & $50,9(44,8-57,0)$ & 37 & $13,7(9,8-18,3)$ & 29 & $10,7(7,3-15,0)$ & 11 & $4,1(2,0-7,1)$ \\
\hline \multicolumn{10}{|l|}{ Raça/Cor da pele ** } \\
\hline Negra (preta + parda) & 369 & 154 & $41,7(36,7-47,0)$ & 119 & $32,2(27,6-37,3)$ & 31 & $8,4(5,9-11,8)$ & 8 & $2,2(1,0-4,4)$ \\
\hline Branca & 132 & 60 & $45,5(36,8-54,3)$ & 41 & $31,1(23,3-39,7)$ & 16 & $12,1(7,1-18,9)$ & 8 & $6,1(2,7-11,6)$ \\
\hline \multicolumn{10}{|l|}{ Local de ocorrência ** } \\
\hline Residência & 286 & 108 & $37,8(32,1-43,7)$ & 144 & $50,3(44,4-56,3)$ & 33 & $11,5(8,1-15,8)$ & 11 & $3,8(1,9-6,8)$ \\
\hline Via pública & 93 & 51 & $54,8(44,2-65,2)$ & 5 & $5,4(1,8-12,1)$ & 7 & $7,5(3,1-14,9)$ & 3 & $3,2(0,7-9,1)$ \\
\hline Escola & 90 & 45 & $50,0(39,3-60,7)$ & 5 & $5,6(1,8-12,5)$ & 5 & $5,6(1,8-12,5)$ & - & - \\
\hline \multicolumn{10}{|l|}{ Relação do provável autor } \\
\hline \multicolumn{10}{|c|}{ da agressão com a vítima ** } \\
\hline Familiar & 189 & 81 & $42,9(35,7-50,2)$ & 91 & $48,1(40,8-55,5)$ & 27 & $14,3(9,6-20,1)$ & 11 & $5,8(2,9-10,2)$ \\
\hline Conhecido & 176 & 97 & $55,1(47,4-62,6)$ & 18 & $10,2(6,2-15,7)$ & 16 & $9,1(5,3-14,3)$ & 2 & $1,1(0,1-4,0)$ \\
\hline Desconhecido & 49 & 24 & $49,0(34,4-63,7)$ & 1 & $2,0(0,1-10,9)$ & 5 & $10,2(3,4-22,2)$ & 4 & $8,2(2,3-19,6)$ \\
\hline \multicolumn{10}{|l|}{ Sexo do provável autor da } \\
\hline \multicolumn{10}{|l|}{ agressão ** } \\
\hline Masculino & 249 & 131 & $52,6(46,2-58,9)$ & 27 & $10,8(7,3-15,4)$ & 31 & $12,4(8,6-17,2)$ & 15 & $6,0(3,4-9,7)$ \\
\hline Feminino & 121 & 51 & $42,1(33,2-51,5)$ & 57 & $47,1(38,0-56,4)$ & 14 & $11,6(6,5-18,7)$ & 1 & $0,8(0,0-4,5)$ \\
\hline Ambos & 26 & 11 & $42,3(23,4-63,1)$ & 19 & $73,1(52,2-88,4)$ & 2 & $7,7(0,9-25,1)$ & - & - \\
\hline Total & 519 & 219 & $42,2(37,9-46,6)$ & 166 & $32,0(28,0-36,2)$ & 49 & $9,4(7,1-12,4)$ & 18 & $3,5(2,1-5,5)$ \\
\hline
\end{tabular}

* Era permitido informar mais de um tipo de violência para a mesma vítima;

** Excluídos os registros sem informação para as variáveis: raça/cor da pele (18), local de ocorrência (50), relação do provável autor da agressão com a vítima (105), sexo do provável autor da agressão (123).

Fonte: Sistema de Serviços Sentinelas de Vigilância de Violências e Acidentes (VIVA), Ministério da Saúde.

A literatura 10,14,15,32 aponta variações quanto ao tipo de agressor conforme o tipo de violência infligida. As maiores prevalências de negligência são praticadas por mãe e pai; a violência psicológica e violência física pela madrasta, o padrasto e outros familiares; e a violência sexual e abuso sexual são mais praticados pelo padrasto e outros familiares.

É necessário apontar algumas limitações do presente estudo, destacando-se a utilização da estratégia de serviços sentinelas, o que não permite obter estimativas populacionais. Além disso, não foi possível incluir todas as capitais, haja vista os problemas operacionais locais. A coleta de dados realizada em um mês para cada serviço pode ter resultado em subnotificação de eventos, além da dificuldade na identificação e mensuração dos casos de abusos sexuais e psicológicos em crianças, especialmente quando cometidos pelos familiares. Por outro lado, a estratégia de serviços sentinelas agrega vantagens como: melhor capacitação e sensibilização da equipe para a realização do inquérito, articulação destes serviços com a rede de proteção à vítima de violência, possibilitando ações integradas e respostas às vítimas, além de disponibilizar informações úteis para o planejamento de medidas de prevenção.

Tais resultados têm sido utilizados no nível local para o melhor conhecimento das violência infligidas contra as crianças e têm mudado a forma de atuar dos serviços notificadores e de toda a 
rede de referência, articulando ações de promoção à saúde, prevenção e controle de violência. A ficha de notificação de violência tem sido cada vez mais adotada pelos municípios e serviços, possibilitando assim a melhor compreensão de uma realidade pouco conhecida.

No Brasil, o fenômeno da violência tem mobilizado as diferentes áreas do conhecimento, bem como o estabelecimento de parcerias visando ao estabelecimento de diferentes estratégias de prevenção e intervenção, no enfrentamento do problema 34,35 . Essa mobilização visa a assegurar o cumprimento de princípios legalmente assegurados no Estatuto da Criança e do Adolescente 23, no que se refere às políticas e programas voltados à violência social e interpessoal contra crianças e adolescentes.

No setor de saúde destacam-se a Política Nacional de Redução da Morbimortalidade por Acidentes eViolências (Portaria ${ }^{\circ}$. 737/2001), a Política Nacional de Promoção da Saúde (Portaria $n^{\circ}$. 687/2006), os instrumentos como a notificação de violência contra crianças e adolescentes na rede assistencial do SUS (Portaria $n^{\circ}$. 1.968/2001), o VIVA e a Rede Nacional de Prevenção da Violência, Promoção da Saúde e Cultura de Paz, contando com núcleos nos estados e municípios (Portaria $n^{o}$. 936/2004) 19,20,24,25,34.

A rede de atenção integral às vítimas de violência vem se consolidando e garantindo o acesso aos serviços de saúde especializados para crianças e adolescentes. Torna-se importante universalizar a notificação dos casos de violência, garantindo o sigilo e orientando a vítima com relação aos seus direitos, bem como o encaminhamento dos casos que necessitem de atendimento específico ou acompanhamento das redes intersetoriais e sociais. Os dados aqui apresentados, coletados pelo VIVA, permitem a identificação dos principais tipos de violência, sua ocorrência, perfil do agressor, dentre outras informações, orientando o desenvolvimento de medidas de intervenção.

\section{Resumo}

O objetivo deste artigo foi descrever o perfil dos atendimentos de emergência por lesões relacionadas à violência em crianças (< 10 anos de idade) atendidas em serviços do Sistema de Vigilância de Violências e Acidentes (VIVA) do Ministério da Saúde, durante 30 dias consecutivos em cidades selecionadas no Brasil, 2006 e 2007. Das 518 crianças atendidas, predominaram as vítimas do sexo masculino (60,6\%), idade de 5-9 anos (52,1\%) e negros (71,2\%). Sobressaíram-se as ocorrências no domicílio (55\%), com lesão por cortel perfuração (34,2\%) e evolução para alta $(68,7 \%)$. A violência mais freqüente foi agressão física $(67,4 \%)$, envolvendo espancamento, objetos perfurocortantes e arma de fogo. Os demais tipos de violência incluíram negligência (32\%), abuso psicológico (9,5\%) e violência sexual (3,5\%). Em sua maioria, os agressores eram do sexo masculino $(48,1 \%)$ e familiares da vítima (36,3\%). Revela-se a vulnerabilidade da criança às situações de violência. É necessário desenvolver estratégias específicas de assistência intersetorial e mobilização social para a intervenção sobre esse problema.

Violência; Criança; Serviços Médicos de Emergência 


\section{Colaboradores}

M. D. M. Mascarenhas contribuiu com a concepção do projeto, organização do banco de dados e elaboração da versão inicial do texto. D. C. Malta e M. M. A. Silva participaram da concepção do projeto, organização do banco de dados e elaboração da versão inicial do texto. C. M. Lima, M. G. O. Carvalho e V. L. A. Oliveira contribuíram na análise dos resultados, discussão e revisão do texto.

\section{Referências}

1. World Health Organization. World report on violence and health. Geneva: World Health Organization; 2002.

2. World Health Organization. World report on child injury prevention. Geneva: World Health Organization/United Nations Children's Fund; 2008.

3. Sanchez RN, Minayo MCS. Violência contra crianças e adolescentes: questão histórica, social e de saúde. In: Ministério da Saúde, organizador. Violência faz mal à saúde. Brasília: Ministério da Saúde; 2006. p. 29-38.

4. Deslandes SF, Assis SG, Santos NC. Violência envolvendo crianças no Brasil: um plural estruturado e estruturante. In: Ministério da Saúde, organizador. Impacto da violência na saúde dos brasileiros. Brasília: Ministério da Saúde; 2005. p. 43-77.

5. Oliveira BRG, Thomazine AM, Bittar DB, Santos FL, Silva LMP, Santos RL, et al. A violência intrafamiliar contra a criança e o adolescente: o que nos mostra a literatura nacional. REME Rev Min Enferm 2008; 12:547-56.

6. Westphal MF, organizador. Violência e criança. São Paulo: Edusp; 2002.
7. Alexandre DT, Vieira ML. Relação de apego entre crianças institucionalizadas que vivem em situação de abrigo. Psicol Estud 2004; 9:207-17.

8. World Health Organization. Preventing child maltreatment: a guide to taking action and generating evidence. Geneva: World Health Organization; 2006.

9. Dahlberg LL, Krug EG. Violence a global public health problem. Ciênc Saúde Coletiva 2006; 11 Suppl:277-92.

10. Costa COM, Carvalho RC, Bárbara JFRS, Santos CAST, Gomes WA, Sousa HL. O perfil da violência contra crianças e adolescentes, segundo registros de Conselhos Tutelares: vítimas, agressores e manifestações de violência. Ciênc Saúde Coletiva 2007; 12:1129-41.

11. Bazon MR. Violências contra crianças e adolescentes: análise de quatro anos de notificações feitas ao Conselho Tutelar na Cidade de Ribeirão Preto, São Paulo, Brasil. Cad Saúde Pública 2008; 24: 323-32. 
12. Carvalho ACR, Barros SG, Alves AC, Gurgel CA. Maus-tratos: estudo através da perspectiva da delegacia de proteção à criança e ao adolescente em Salvador, Bahia. Ciênc Saúde Coletiva 2009; 14:539-46

13. Moura ATMS, Reichenheim ME. Estamos realmente detectando violência familiar contra a criança em serviços de saúde? A experiência de um serviço público do Rio de Janeiro, Brasil. Cad Saúde Pública $2005 ; 21: 1124-33$.

14. Gomes MLM, Falbo Neto GH, Viana CH, Silva MA Perfil clínico-epidemiológico de crianças e adolescentes do sexo feminino vítimas de violência atendidas em um Serviço de Apoio à Mulher, Recife, Pernambuco. Rev Bras Saúde Matern Infant 2006; 6 Suppl 1:S27-34.

15. Monteiro CFS, Teles DCBS, Castro KL, Vasconcelos NSV, Magalhães RLB, Deus MCBR. Violência sexual contra criança no meio intrafamiliar atendidos no SAMVVIS, Teresina, PI. Rev Bras Enferm 2008; 61:459-63.

16. Ferreira AL. Acompanhamento de crianças vítimas de violência: desafios para o pediatra. J Pediatr (Rio J.) 2005; 81 Suppl:S173-80.

17. Nunes CB, Sarti CA, Ohara CVS. Conceptions held by health professionals on violence against children and adolescents within the family. Rev Latinoam Enferm 2008; 16:136-41.

18. Concha-Eastman A, Clavel-Arcas C. Injuries from external causes: progress in data management, analysis, and patient care. Rev Panam Salud Pública 2008 ; $24: 375-8$.

19. Gawryszewski VP, Silva MMA, Malta DC, Mascarenhas MDM, Costa VC, Matos SG, et al. A proposta da rede de serviços sentinela como estratégia de vigilância de violências e acidentes. Ciênc Saúde Coletiva 2006; 11 Suppl:1269-78.

20. Portaria $n^{\circ}$. 737. Dispõe sobre a Política Nacional de Redução da Morbimortalidade por Acidentes e Violências. Diário Oficial da União 2001; 18 mai.

21. Cavalcante FG, Goldson E. Situational analysis of poverty and violence among children and youth with disabilities in the Americas - an agenda proposal. Ciênc Saúde Coletiva 2009; 14:7-20.

22. Deslandes SF, Lemos MP. Construção participativa de descritores para avaliação dos núcleos de prevenção de acidentes e violência, Brasil. Rev Panam Salud Pública 2008; 24:441-8.
23. Lei $n^{\circ}$. 8.069. Dispõe sobre o Estatuto da Criança e do Adolescente, e dá outras providências. Diário Oficial da União 1990; 16 jul.

24. Malta DC, Silva MMA, Mascarenhas MDM, Souza MFM, Morais Neto, Costa VC, et al. A vigilância de violências e acidentes no Sistema Único de Saúde: uma política em construção. Divulg Saúde Debate 2007; 39:82-92.

25. Portaria $n^{\circ}$. 936. Dispõe sobre a estruturação da Rede Nacional de Prevenção da Violência e Promoção da Saúde e a Implantação e Implementação de Núcleos de Prevenção à Violência em Estados e Municípios. Diário Oficial da União 2004; 20 mai.

26. Ricas J, Donoso MTV, Gresta MLM. A violência na infância como uma questão cultural. Texto \& Contexto Enferm 2006; 15:151-4.

27. Souza ER. Masculinidade e violência no Brasil: contribuições para a reflexão no campo da saúde. Ciênc Saúde Coletiva 2005; 10:59-70.

28. Silva MAI, Ferriani MGC. Domestic violence: from the visible to the invisible. Rev Latinoam Enferm 2007; 15:275-81.

29. Tavares ML. Abordagem da violência intrafamiliar no programa saúde da família. In: Ministério da Saúde, organizador. Violência faz mal à saúde. Brasília: Ministério da Saúde; 2006. p. 205-18.

30. Filócomo FRF, Harada MJS, Silva CV, Pedreira MLG. Estudo dos acidentes na infância em um prontosocorro pediátrico. Rev Latinoam Enferm 2002; 10:41-7.

31. Martins CBG. Acidentes na infância e adolescência: uma revisão bibliográfica. Rev Bras Enferm 2006; 59:344-8.

32. Faleiros JM, Matias ASA, Bazon MR. Violência contra crianças na cidade de Ribeirão Preto, São Paulo, Brasil: a prevalência dos maus-tratos calculada com base em informações do setor educacional. Cad Saúde Pública 2009; 25:337-48.

33. Minayo MCS. A inclusão da violência na agenda da saúde: trajetória histórica. Ciênc Saúde Coletiva 2006; 11 Suppl:1259-67.

34. Silva MMA, Malta DC, Morais Neto OL, Rodrigues EMS, Gawryszewski VP, Matos S, et al. Agenda de prioridades da vigilância e prevenção de acidentes e violências aprovada no I Seminário Nacional de Doenças e Agravos Não Transmissíveis e Promoção da Saúde. Epidemiol Serv Saúde 2007; 16: 57-64

Recebido em 19/Ago/2009

Versão final reapresentada em 28/Out/2009

Aprovado em 01/Dez/2009 